\title{
Duchenne muscular dystrophy presenting with failure to thrive
}

\author{
R Rapisarda, F Muntoni, P Gobbi, V Dubowitz
}

\begin{abstract}
Three patients with Duchenne muscular dystrophy presenting with failure to thrive in infancy are described. Their families noted poor growth and sought medical advice up to six months before the diagnosis was suspected. Growth charts showed weight loss between 4 and 18 months of age. Length growth was affected while growth in head circumference remained unaffected. No causes of failure to thrive were found at presentation. Failure to thrive in Duchenne muscular dystrophy has been described previously but it is neither widely recognised nor understood. Poor growth in the first years and eventual short stature, however, is a well described feature of Duchenne muscular dystrophy. Failure to thrive in our three patients may be an extreme manifestation of this.

(Arch Dis Child 1995; 72: 437-438)
\end{abstract}

Keywords: muscular dystrophy, failure to thrive, growth, dog diseases.

Duchenne muscular dystrophy is the most common lethal $\mathrm{X}$ linked disease in man, with an incidence of approximately one in 3500 male births. It usually presents clinically between 3 and 5 years of age because of difficulties with locomotion. An early presentation with failure to thrive has been described by Call and Ziter in five patients presenting between 5 and 15 months of age. ${ }^{1}$ Three further patients are reported here.

\section{Case reports}

CASE 1

The birth and neonatal period of this boy were normal; the birth weight was $3690 \mathrm{~g}$ (50th centile), length $51 \mathrm{~cm}$ (50th centile), and head circumference $34 \mathrm{~cm}$ (10-50th centile). His parents first became concerned at 6 months of age because of poor weight gain. His weight fell from the 50 th centile at birth to the 3 rd centile by 9 months. Length showed a similar decrease, while head growth was not affected.

No obvious cause was found to explain the failure to thrive. Energy intake appeared to be adequate and his parents reported no overt illness nor difficulty with feeding. Physical examination at 10 months showed mild calf hypertrophy, head lag when pulled to sit, and laxity of the wrists and ankles. He was unable to sit unsupported and only acquired this ability at 11 months. He was able to commando crawl at 16 months. At that time he was not yet saying any words.

Duchenne muscular dystrophy was suspected at 9 months because of delayed motor milestones and a raised creatine kinase of 7130 $\mathrm{U} / 1$ (normal $<200 \mathrm{U} / \mathrm{l}$ ) and confirmed on muscle histology, dystrophin immunocytochemistry and western blot, which showed complete absence of dystrophin. No deletion was found on polymerase chain reaction multiplex DNA analysis.

\section{CASE 2}

The birth and neonatal period of this boy were uneventful; the birth weight was $3660 \mathrm{~g}$ (50th centile) and head circumference $34.5 \mathrm{~cm}$ (10-50th centile). The parents first became concerned at 1 year of age because of poor weight gain, his weight having fallen from the 50 th centile to below the 10 th. Head circumference was unaffected (10-50th centile). Delayed motor milestones were noted at this time but not investigated. At 18 months he was investigated for failure to thrive and no cause was found. An incidental creatine kinase was grossly raised.

Developmental milestones showed global delay. He crawled at 12 months, sat at 18 months, stood unsupported at 20 months, walked and only used single words at 23 months. On examination at 23 months he had a normal gait but was unable to hop and required support to rise from the floor. Hypertrophy of the vastus lateralis was evident.

Duchenne muscular dystrophy was suspected at 18 months because of the developmental delay and raised creatine kinase and was confirmed by muscle histology, dystrophin deficiency on immunocytochemistry, and out of frame deletion of exons $45-50$ on DNA analysis.

\section{CASE 3}

This boy presented at 6 months of age because of failure to gain weight over the previous two months. He had a normal delivery, his birth weight was $3030 \mathrm{~g}$ (10th centile), length 51.5 cm (50-90th centile), and head circumference $34 \mathrm{~cm}$ (10th centile).

He was fed with formula milk from birth and showed weight gain along the 50th centile at 4 months of age. He was admitted at 6 months of age for dehydration associated with diarrhoea and failure to gain weight for the previous two months. His weight had fallen from the 50th centile to below the third at 6 months. His length was similarly affected while head 
circumference showed no fall off. Extensive investigations for failure to thrive including jejunal biopsy were unremarkable, but the creatine kinase on biochemical screening was raised at $7505 \mathrm{U} / \mathrm{l}$.

Global developmental delay later became apparent. He sat at 11 months, could say a few syllables at 12 months, and walked independently at 24 months. Examination at 12 months showed normal muscle bulk, and normal tone and reflexes. He had at least antigravity power in the limbs, but was unsteady while sitting and had poor head control.

Duchenne muscular dystrophy was suspected at 7 months because of the raised creatine kinase value. Muscle histology showed dystrophic changes with a very reduced amount of dystrophin on immunocytochemistry staining. A Duchenne muscular dystrophy deletion study showed an in-frame deletion of exons $62-63$.

\section{Discussion}

These three patients presented with failure to thrive between 4 months and 18 months of age. There was corresponding slowing in length growth in the two patients ( 1 and 3 ) for whom data were available. Head circumference remained unaffected in all three cases. Diagnosis of Duchenne muscular dystrophy was delayed for up to six months after presentation with failure to thrive. Considering the three patients had no family history of the disease, an early diagnosis might have had important implications for genetic counselling. Delayed motor milestones and physical signs of Duchenne muscular dystrophy, weakness and muscle enlargement, were apparent at, or within a few months of, presentation.

Growth studies of large groups of boys with Duchenne muscular dystrophy have found normal weights and lengths at birth and subsequent slowing of growth during the first years of life. After the age of 3 years normal growth patterns resume with velocities appropriate for age though following lower centiles. These changes were more marked for height compared with weight while bone maturation was unaffected. ${ }^{2} 3$ No adequate explanation has been found for the short stature of Duchenne muscular dystrophy. It is unlike the growth pattern in chronic diseases which is associated with delayed bone maturation and catch up growth to previous centiles.

Pathological changes seen on muscle biopsy specimens have been described in the early stages of Duchenne muscular dystrophy even before overt clinical signs appear. ${ }^{4}$ Muscle cell regeneration is most prominent at this early stage $^{5}$ and blood values of creatine kinase, which reflect muscle necrosis, reach a peak during this period. ${ }^{6}$

Similar findings are present in the canine $\mathrm{X}$ linked muscular dystrophy (CXMD) dog, which is an animal model for Duchenne muscular dystrophy. Due to a mutation in the homologue of the Duchenne muscular dystrophy locus, affected dogs are dystrophin deficient and have morphological similarities to Duchenne muscular dystrophy. Apart from a fulminant form of CXMD which is fatal in the neonatal period, the clinical course is analogous to Duchenne muscular dystrophy. Observations of growth in 14 dogs with CXMD by Valentine et al found no difference in body weight and crown-rump length at birth between normal and affected dogs. ${ }^{7}$ Surviving dogs had poor growth in the first six weeks of life before any clinical signs of neuromuscular disease. Histological changes of muscle necrosis and regeneration and raised creatine kinase values are also present in CXMD before the onset of clinical signs. ${ }^{8}$ From these studies it seems that early poor growth in Duchenne muscular dystrophy and CXMD coincides with a period of intense muscle necrosis and regeneration and may be due to increased metabolic activity. Failure to thrive may be an extreme manifestation of growth retardation found in most patients with the disease. Assessment of energy intake and basal metabolic rate in subjects with CXMD and Duchenne muscular dystrophy during this period would help to clarify this phenomenon.

We are grateful to Drs S Hodgson and R Roberts, Guy's Hospital, St Thomas' Street, London for the results of polymerase chain reaction multiplex DNA analyses.

Dr R Rapisarda is a trainee clinical fellow supported by the Dr R Rapisarda is a trainee clinical fellow
Muscular Dystrophy Group of Great Britain.

1 Call G, Ziter FA. Failure to thrive in Duchenne muscular dystrophy. F Pediatr 1985; 106: 939-41.

2 Eiholzer U, Bolthauser E, Frey D, Molinari L, Zachmann M. Short stature: a common feature in Duchenne muscular dystrophy. Eur $¥$ Pediatr 1988; 147: 602-5.

3 Rapaport D, Colletto G, Vainzof M, Duaik MC, Zatz M. Short stature in Duchenne muscular dystrophy. Growth Regulation 1991; 1: 11-5.

4 Dubowitz V. The muscular dystrophies. In: Dubowitz V, ed. Muscle biopsy: a practical approach. 2nd Ed. London: Ballière Tindall, 1985: 289-403.

5 Walton JN. Progressive muscular dystrophy: structural alterations in various stages and in carriers of Duchenne dystrophy. In: Pearson CM, Mostofi FK, eds. The striated muscle. London: Williams and Wilkins, 1973: 263-91.

6 Pennington RJT. Clinical biochemistry of muscular dystrophy. Br Med Bull 1980; 36: 123-6.

7 Valentine BA, Cooper BJ, de Lahunta A, O'Quinn R, Blue JT. Canine X-linked muscular dystrophy, an animal model of Duchenne muscular dystrophy: clinical studies. $\mathcal{I}$ Neurol Sci 1988; 88: 69-81.

8 Valentine BA, Cooper BJ, Cummings JF, de Lahunta A. Canine $\mathrm{X}$-linked muscular dystrophy: morphologic lesions. f Neurol Sci 1990; 97: 1-23. 